\title{
Molecular Origins of Elastomeric Friction
}

\author{
Scott Sills, ${ }^{1,3}$ Katherine Vorvolakos, ${ }^{2}$ Manoj K. Chaudhury, ${ }^{2}$ René M. Overney ${ }^{1}$ \\ ${ }^{1}$ Department of Chemical Engineering, University of Washington, Seattle, WA 98195; ${ }^{2}$ Department of Chemical Engineering, \\ Lehigh University, Lehigh, PA, ${ }^{3}$ IBM Almaden Research Center, 550 Harry Road, San Jose, CA 95120
}

\begin{abstract}
Frictional properties of soft elastomers have been in question for over half of a century. Early studies $^{1-3}$ on natural rubber originated for the sole purpose of tabulating properties for bulk consumer applications, such as viscoelastic adhesives, ${ }^{4,5}$ tires, ${ }^{6}$ and windshield wipers, ${ }^{7,8}$ to name a few. Empirical tabulation of frictional properties persisted until the early 1950's, when Roth et al. $^{9}$ and Thirion ${ }^{10}$ began experiments towards a fundamental understanding of rubbery sliding. Quantitative physical analysis began with the observation that the classic Coulombic laws obeyed consistently at rigid body interfaces fail at the interface between a rigid solid and a rubber. Even today, there remains an incomplete understanding of the molecular level parameters that control the frictional behavior of elastomeric surfaces. With this chapter, we explore the historical developments in elastomeric friction and discuss the evolution of an unresolved triborheological complexity. We work from an initial macroscopic perspective toward a microscopic one that describes dissipation process in terms of molecular phenomena at frictional contacts. Readers are urged to consider a competition between these molecular processes, where for soft matter, internal cohesion is comparable to interfacial adhesion. While cohesion may dominate adhesion, or visa versa, we develop a picture for elastomeric friction that encompasses both.
\end{abstract}

\section{Early Elastomeric Friction Studies}

Early studies by Papenhuyzen, ${ }^{11}$ as well as Roth et al.., ${ }^{9}$ showed that the friction force of commercial rubbers on steel increases monotonically with velocity. Beyond a certain velocity, however, sliding becomes unstable and the rubber sample "chatters", or exhibits stick-slip sliding. Thirion ${ }^{10}$, on the other hand, observed that the friction increases with normal load, which Schallamach ${ }^{12,13}$ attributed to the increase of contact area resulting from the deformation of rubber asperities. Similar suggestions were made by Bowden and Tabor. ${ }^{14}$ Assuming the asperities are hemispheres in Hertzian contact with smooth glass, Schallamach predicted that friction should increase with load in a power law manner, with an exponent of two third. Indeed, this prediction was verified over a limited range of loads. However, Schallamach did not immediately address a crucial implication of his prediction: that the friction force should increase with modulus! If frictional force were to depend on contact area, a softer (lower modulus) material would have a greater contact area at any load, thus exhibiting higher friction, which was contrary to several experimental observations. He moved on to examine the effects of velocity and temperature ${ }^{19}$ on rubber friction. As temperature increases, the frictional force decreases. Alternatively, at a given temperature, the friction force increases with sliding velocity. Schallamach showed that the velocity- and temperature-dependent behavior of rubber friction follows Eyring's ${ }^{15}$ theory of reaction rates. However, when this activation theory is applied to 
explain elastomeric friction, interfacial sliding can be described by both tribological (interfacial) and rheological (bulk material) models. While the formation and breakage of adhesive molecular bonds occur at the contact interface, viscoelastic molecular relaxation of the stressed molecules constitutes an internal friction component within the bulk elastomer, i.e. the friction associated with the molecules sliding along themselves. Resolving the respective contribution of each dissipative process to the overall friction remains an ongoing challenge that represents the triborheological complexity.

While Schallamach focused on the molecular processes at the interface, Greenwood and Tabor $^{16}$ as well as Bueche and Flom ${ }^{17}$ pointed out that the energy of sliding a soft viscoelastic material over a rigid substrate is not spent entirely in breaking molecular contacts at the interface, but at least partially on deforming the soft material. The notion that friction might be a combination of surface and bulk effects prompted $\operatorname{Grosch}^{18}$ to perform the most systematic study in the field to date. He measured the effects of velocity, temperature, and surface roughness for rubber sliding on optically smooth glass. Grosch observed that the rubber friction increases nonlinearly with velocity, much like the shear thinning behavior of high viscous polymers. Above a certain critical velocity, the friction force exhibits a stick-slip behavior with the maximum friction in each pulse decreasing with velocity. Furthermore, at each sliding velocity, friction decreases with increasing temperature. Grosch collapsed his friction-rate isotherms into a single master curve using the well-known Williams, Landel and Ferry ${ }^{19}$ (WLF) superposition principle, which is pervasive in bulk rheology. His most intriguing finding was that the velocity corresponding to maximum friction and the frequency corresponding to maximum viscoelastic loss form a ratio that is nearly constant $(\sim 7 \mathrm{~nm})$ for various rubbery materials. He asserted that the interfacial relaxation processes responsible for friction are related to the segmental relaxation of polymer chains. With this, he initiated a debate that continues today: whether the origin of elastomeric friction lies in interfacial adhesion or viscous relaxation of the bulk elastomer.

The critical length of $7 \mathrm{~nm}$ represents a molecular length, presumably the characteristic length for molecular jumps in an adhesive picture for sliding friction, or alternatively, the length scale for viscoelastic relaxation in the bulk elastomer. For rough surfaces, the relevant length scale was found to be the characteristic spacing between surface asperities. Grosch's general observations of the dependence of friction on velocity and temperature were also supported by Extrand et al. ${ }^{8}$, who examined the more practical geometry of sharp rubber edges against rigid surfaces. Extrand et al. noted that the coefficient of friction depends strongly on the local load and the results are dependent on the surface preparation, i.e. chlorination of natural rubber, which may effect both the viscoelastic moduli and the interfacial adhesion.

Prompted by Grosch's observations, Schallamach ${ }^{20}$ refined his model of interfacial friction, since a prediction of a monotonic dependence of friction on velocity was insufficient. He maintained that unlubricated sliding on smooth surfaces is essentially adhesive in nature, mediated by separate bonding and debonding events between the rubber and the rigid surface, depicted in Figure 1. 


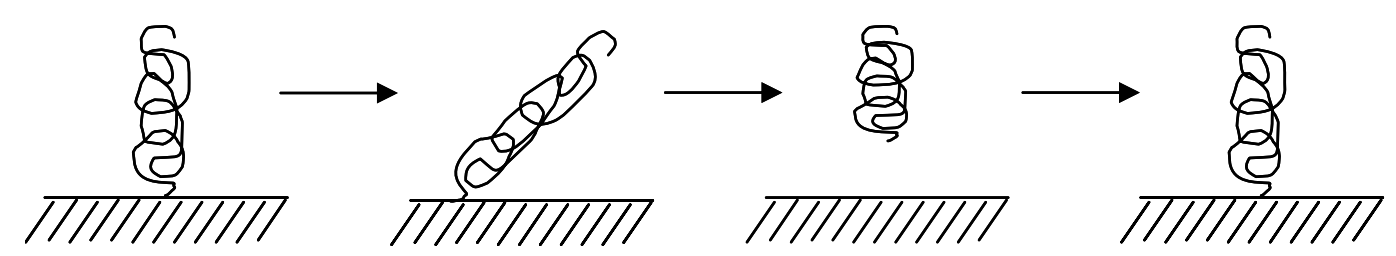

Figure 1. The classic depiction of a polymer chain in contact with a laterally moving countersurface. The chain stretches, detaches, relaxes and re-attaches to the surface to repeat the cycle. Adapted from reference ${ }^{21}$.

Schallamach's ${ }^{20}$ explanation of Grosch's ${ }^{18}$ observations was based on the rate-dependent molecular debonding model of Frenkel ${ }^{22}$ and Eyring ${ }^{15}$. In this model, the probability of debonding a polymer chain from a surface is a product of two functions, the first being the frequency factor that increases exponentially with the applied force, and the second being the number of load-bearing chains that decreases with velocity. The solution of the kinetic rate equation resulting from such considerations, leads to an expression for the debonding force that increases with velocity, while the number of the load-bearing polymer chains $(\Sigma)$ decreases (Figure 2). The net effect is that the total interfacial stress at first increases with velocity, reaches a maximum, and thereafter decreases with velocity.

\section{Stochastic Adhesive Model}

A couple of decades ago, Chernyak and Leonov ${ }^{23}$ refined Schallamach's picture of rubber friction by using a steady state stochastic model for debonding kinetics. Within this model, directional stretching of polymer chains occurs as a result of an external force, leading to the detachment of linking chains from the wall. A newly detached chain dissipates the elastic energy accumulated during stretching and re-attaches to the surface. By considering the stochastic nature of detachment force, Chernyak and Leonov ${ }^{23}$ derived the shear stress in dry sliding as given by Equation 1,

$$
\sigma_{t}=\Sigma_{\circ} \frac{\int_{0}^{\infty} \varphi\left(\frac{r(t)}{\delta}\right) p(V, t) d t}{V\left[\langle t\rangle_{b}+\langle t\rangle_{f}\right]}
$$

where $\Sigma_{\mathrm{o}}$ is the areal density of the load bearing chains at zero velocity, $\varphi\left(\frac{r(t)}{\delta}\right)$ is the elastic energy stored in the stretched polymer chain, $V$ is the sliding velocity, $\langle t\rangle_{b}$ is the mean lifetime of contact, $\langle t\rangle_{f}$ is the time the polymer chain spends in free state, $p(V, t)$ is the transition probability of the polymer chain in going from the bonded to the debonded, elastically restored state. The numerator of the Chernyak and Leonov equation (Equation 1) is the work done in stretching the polymer chain to the detachment point, while the denominator represents the mean distance traveled by the chain. Multiplication of this stochastic force with the areal density of the linking chains gives rise to the expression for 
shear stress. This formulation however, neglects the fact that polymers are not perfectly elastic and can dissipate energy during the stretching process, through the viscous modes associated with internal friction.

Using a steady state stochastic model of bond dissociation, Chernyak and Leonov showed that the mean lifetime of contact $\langle t\rangle_{b}$ and the transition probability depend on the sliding velocity as shown, respectively, in Equations 2 and 3.

$$
\begin{aligned}
& \langle t\rangle_{b}=\tau_{\circ}\left\{1-\exp \left(-\frac{V}{V_{\circ}}\right)\right\} \\
& p(V, t)=\exp \left(-\frac{t}{\tau_{\circ}}\right)\left\{\delta\left(t-t_{b}\right)-\frac{\theta\left(t_{b}-t\right)}{\tau_{\circ}}\right\}
\end{aligned}
$$

Here, $\delta(z)$ represents Dirac's delta function corresponding to the determinate process of forced break-off, and $\theta(\mathrm{z})$ is the Heaviside step function. With the above definitions of the bond lifetime and the transition probability, Equation 1 can be integrated for simple Gaussian polymer chains, the elastic energy of which is proportional to the square of the extension. Shear stress can then be expressed as follows:

$$
\sigma=\sigma_{a}(m+1) \frac{u(1+s)\left(1-\exp \left(\frac{-1}{u}\right)-\exp \left(\frac{-1}{u}\right)\right)}{m+1-\exp \left(\frac{-1}{u}\right)}
$$

where $m$ is the fundamental ratio of the lifetimes of the polymer chain in the free and bound states at zero sliding velocity, $s$ is the ratio of the viscous retardation time over the lifetime at rest and $u$ is the dimensionless velocity of sliding defined by Equation 5,

$$
u=\frac{V \tau_{\circ}}{\delta a}
$$

where $\tau_{o}$ is the lifetime of the bound state at rest, $\delta$ is the average distance between the polymer body and the wall, and $a$ is the statistical segment length of the polymer. $\sigma_{a}$ is defined by Equation 6 .

$$
\sigma_{a}=\frac{k T \Sigma_{\circ} \delta}{(1+m) R_{F}^{2}}
$$

$R_{F}$ is the Flory radius of the polymer chain. Equation 4 predicts that the shear stress first increases with velocity in an $s$-shaped manner. After exhibiting a rather broad maximum, $\sigma$ usually decreases at very high sliding velocities. 


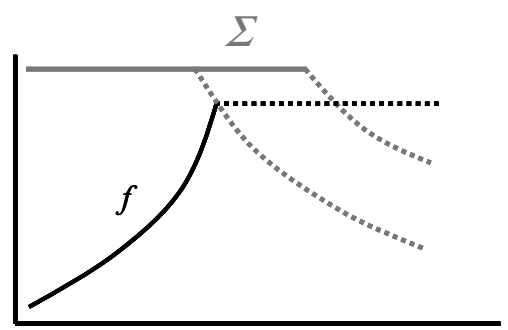

$\log V$

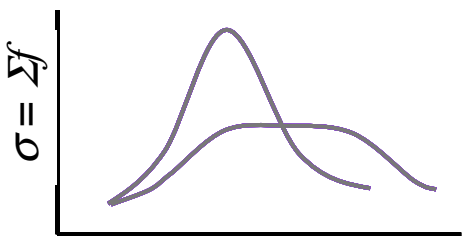

$\log V$

Figure 2. The left figure qualitatively depicts the behavior of the areal density of contact points and the force per adsorption point as a function of velocity. The former decreases, while the latter increases up to a value limited by the interaction strength between the polymer chain and the countersurface. The product of these two quantities yields the shear stress, which increases and subsequently decreases, depicted on the right. Adapted from reference ${ }^{21}$.

\section{Viscoelastic Perspective: Contributions from internal friction}

Schallamach $^{20}$ and Chernyak and Leonov $^{23}$ developed their models envisioning purely adhesive sliding. However, Savkoor ${ }^{24}$ as well as Ludema and Tabor $^{25}$ suggested that even seemingly adhesive sliding could never be purely adhesive. Savkoor ${ }^{24}$ proposed a hybrid model, in which the interface consists of discrete patches of asperities of molecular dimensions in adhesive contact with the rubber surface. When a shear force is imposed, the patch stores elastic energy until it overcomes the adhesive energy, causing the propagation of a shear crack. Again, these early models neglected the inherent viscous attributes of elastomers. According to Savkoor ${ }^{28}$ as well as Ludema and Tabor, ${ }^{25}$ sliding may proceed by an activated process, but the extent to which the two surfaces come into contact depends on modulus and sliding velocity. Hidden in more macroscopic terms, these approaches of Savkoor $^{24}$ and Ludema and Tabor ${ }^{25}$ are similar to the model of Schallamach, ${ }^{20}$ where the activated nature of the sliding process is still attributed to adhesive debonding.

An activated sliding process does not necessarily identify friction as adhesion dominated. A realistic picture of the contact interface must also consider the viscoelastic nature of the elastomer during the shear process, which from a microscopic perspective involves intermolecular sliding. Intermolecular sliding is an activated relaxation process that can also be described with the Erying model discussed above. Its related local force, i.e., internal friction, is strongly intertwined with the cohesive forces within the bulk elastomer. Considering now both, the intermolecular relaxation in the polymer "bulk" system due to shear, and the adhesive bonding-debonding process, elastomeric friction can be considered a tribo-rheological process. If the adhesive force, $F_{a d h}$, exceeds the cohesion force, $F_{c o h}$, the contact undergoes cohesive yield, i.e., "bulk" polymer relaxation properties dictate friction, and visa versa. In addition, it has to be pointed out that the two processes possess specific characteristic time scales. Thus, depending on the experimental time window, i.e., the scan velocity, a loss spectrum analysis based on friction-velocity isotherms can be sensitive to either of the two proceses, or both. 
When the time scale of the driven surface is much slower than the response time of the material, the contact ages prior to a virtual jump of the driven surface. In this regime, friction may actually increase as velocity decreases because the net adhesion force is proportional to the contact aging time. On the other hand, when the scan velocity is much faster than the interface can respond, the surfaces do not easily slide relative to each other. Instead, the surfaces start peeling locally and detachment waves propagate throughout the entire area of contact, from its advancing to the trailing edge. Schallamach ${ }^{26}$ first discovered these waves at high sliding velocities. Roberts and Jackson ${ }^{27}$ suggested that when such instabilities occur, the frictional stress between surfaces can be described in terms of the wavelength $(\Lambda)$ of the Schallamach instability and the adhesion hysteresis $(\Delta W)$, i.e. the energetic difference involved in making and breaking interfacial contacts, as $\sigma=\Delta W / \Lambda$. In the region where the experimental time scale is comparable to the characteristic material times, the measured frictional forces are representative of the relevant dissipative mechanisms. However, identifying the dominant friction mechanism requires some characteristic signature for the dissipation process.

In ambient scanning force microscopy (SFM) experiments and computer models, such as molecular dynamic (MD) and Monte Carlo (MC) simulations, molecular scale friction is discussed in terms of mechanical relaxations and internal conformational changes 28-31. These studies involved highly structured model systems, which were prepared by either self-assembly or Langmuir-Blodgett techniques ${ }^{32}$. Such mono- or multilayered systems provide convenient access for investigations of molecular-scale dissipation mechanisms; e.g. load induced molecular tilts ${ }^{28}$, and reversible and irreversible conformation changes ${ }^{28-30}$. Simple frictional models, such as the Tomlinson-Prandtl model ${ }^{33}$, could be tested, and the corrugated molecular surface potential compared to the magnitude of discontinuous molecular stick-slip sliding ${ }^{29,31}$. The jump-distances were found to be stochastic above a critical sliding velocity, which led to a discussion of molecular friction in terms of fluctuations around discrete attractors ${ }^{31}$. This corresponds to recent theoretical treatments, i.e. creep models that consider barrier-hopping fluctuations of periodic surface potentials with slips occurring at lower energy values than prescribed by the potential barriers.

Sills and Overney ${ }^{37}$ showed that hindered, or frozen, relaxation states of an amorphous polymer could be activated in the course of a frictional sliding process, and thus, give rise to a barrier-hopping fluctuation not unlike the one observed for highly ordered surfaces. Friction-rate isotherms obtained with a SFM tip on glassy polystyrene could be collapsed to a master curve according to a ramped creep model ${ }^{34,35}$, Figure 3 , which considers a single asperity sliding over a corrugated surface potential that is biased due to the motion of the driven tip, i.e. the probability of a backwards jump is much lower than for a forward jump. The barrier height becomes proportional to a 3/2-power law in the friction force $^{34,35}$, which leads to a distorted power-law friction-velocity relationship of $F=F_{c}-\Delta F\left|\ln \left(V^{*}\right)\right|^{2 / 3}$. Based on Sang et al.'s theory, ${ }^{35} V^{*} \sim V / T$ represents a dimensionless velocity, and $\Delta F \sim \mathrm{T}^{2 / 3} . F_{c}$ is an experimentally determined constant that contains the critical position of the cantilever support. $F_{c}$ is determined from the intercept of $F$ versus $T^{2 / 3}$ at a fixed ratio $T / V=1 \mathrm{~K} /(\mathrm{nm} / \mathrm{s})$. 


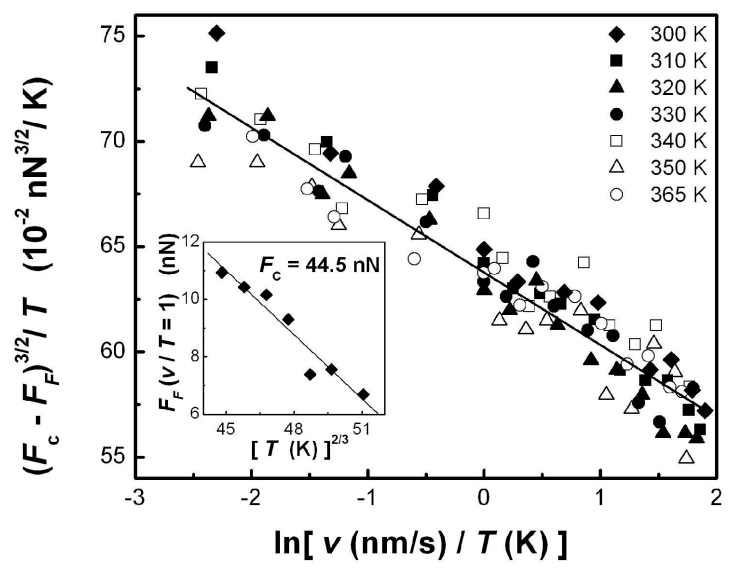

Figure 3. Collapse of poslysyrene friction-rate isotherms to a ramped creep barrier-hopping fluctuation model. (inset) the constant $F_{c}$ is determined from the intercept of $F$ versus $T^{2 / 3}$ for a fixed ratio $T / V=1 \mathrm{~K} /(\mathrm{nm} / \mathrm{s})$. Adapted from reference ${ }^{37}$.

Interestingly, ramped creep scaling is consistent with the solution of the Langevin equation for a perfect cantilever oscillator in the total potential energy $E$

$$
M \ddot{x}+M \beta \dot{x}+\frac{\partial E(x, t)}{\partial x}=\xi(t)
$$

with thermal noise in the form of the random force, $\xi(t)$, where $\left\langle\xi(t) \xi\left(t^{\prime}\right)>=2 M \beta k_{b} T \delta\left(t-t^{\prime}\right), x\right.$ is the position of the tip on the surface, $M$ is the mass of tip, and $\beta$ is a linear dampening factor. Equation 7 is based on a sinusoidal surface potential continuously overcome during the course of frictional sliding, which Sills and Overney experimentally determined as $7 \mathrm{kcal} / \mathrm{mol}$ on glassy polystyrene. ${ }^{37}$ This activated process corresponds directly with the hindered rotation of the phenyl ring side chains about their bond with the backbone, supporting the contribution of intrinsic molecular relaxation to elastomeric friction. In a similar SFM friction study on poly(methyl methacrylate), Hammerschmidt et al.. identified the $\beta$ relaxation of carboxylate side-chains as the primary dissipation mechanism ${ }^{38}$. In these examples, the effect of adhesion between the silicon tip and polystyrene was negligible. However, it is possible that asperities become coated with elastomer molecules, resulting in a cohesive sliding interface that is chemically indistinct from intrinsic molecular relaxation within the bulk elastomer.

\section{A Discussion in the Light of Adhesive Stochastic Theory}

The above models, all of which offer plausible explanations for interfacial friction, have yet to be rigorously tested experimentally. Decoupling the adhesive and cohesive 
(viscoelastic) contributions to elastomeric friction requires comprehensive experimental designs to access the energetic signatures of dissipation mechanisms, and the use of model elastomeric networks and counter-surfaces. To maintain a constant adhesion force, the elastomeric contacts would have to be chemically similar, while the internal friction component is varied by confining segmental relaxation through crosslinking. ${ }^{39}$ On the other hand, holding the cohesive component constant and varying the adhesion requires countersurfaces with different surface energies. Both surfaces have to be free of secondary interactions and robust enough to withstand wide ranges of sliding velocity and temperature, without compromising the ideality of the sliding materials.

Model studies of these types have been initiated by several groups. For example, Brown $^{40}$ and Casoli et al.. ${ }^{41}$ examined the pull-out of polymer chains from elastomeric networks and the associated friction. Ghatak et al. ${ }^{42}$ studied friction of polydimethylsiloxane (PDMS) on some low energy surfaces as a function of molecular weight of the polymer $M$ and the sliding velocity. They noted that friction decreases with molecular weight. However, it should be pointed out that these studies were limited in the range of molecular weight, and irrespective of the critical molecular weight for entanglements $M_{e}$. Vorvolakos and Chaudhury $^{21}$ extended these studies using crosslinked hemispheres of PDMS, in sliding contact with two low energy surfaces: a methyl functional self-assembled monolayer (SAM) of hexadecylsiloxane and a thin film of polystyrene (PS), both of which interact with PDMS via dispersion interactions. Using these simple model systems, they carried out the measurements of adhesion and friction to investigate how the latter depends on surface energy, temperature, velocity, and intercrosslink molecular weight of the elastomer. Contact mechanics measurements in the manner of Johnson, Kendall and Roberts (JKR) yielded the modulus of each network and the works of adhesion when placed in contact with each lowenergy surface, as shown in Table 1.

\begin{tabular}{cccc}
$\begin{array}{c}\mathrm{M} \\
(\mathrm{kg} / \mathrm{mol})\end{array}$ & $\begin{array}{c}\mathrm{G} \\
\left(10^{5} \mathrm{~N} / \mathrm{m}^{2}\right)\end{array}$ & $\begin{array}{c}\mathrm{W}_{\text {PDMS-SAM }} \\
(\mathrm{mJ} / \mathrm{m} 2)\end{array}$ & $\begin{array}{c}\mathrm{W}_{\text {PDMS-PS }} \\
(\mathrm{mJ} / \mathrm{m} 2)\end{array}$ \\
\hline 1.3 & 31.4 & 42 & 53 \\
1.9 & 20.3 & 41 & 55 \\
2.7 & 16.0 & 44 & 56 \\
4.4 & 8.8 & 42 & 53 \\
8.9 & 4.3 & 42 & 52 \\
18.7 & 2.6 & 42 & 44 \\
52.1 & 0.6 & 27 & 26
\end{tabular}

Table 1. Molecular weight, $M$, shear modulus, $G$, and works of adhesion, $W$, for each PDMS network. The strength of interaction is largely independent of $M$ for $M<M_{e} \sim 8 \mathrm{~kg} / \mathrm{mol}$, but drops significantly for $M>M_{e}$, which suggests that the degrees of freedom available to molecular interactions are restricted due to entanglements. ${ }^{21}$.

Friction against each surface was measured over a velocity range spanning $10^{-7}$ to $10^{-3} \mathrm{~m} / \mathrm{sec}$, comparable to the range studied by Reiter et al. ${ }^{41}$. The friction force $F$ divided by the optically measured contact area $A$ yielded the shear stress $\sigma$ reported in Figure 4 . In all cases, the shear stress increases with velocity up to a certain critical velocity $V_{o}$, then plateaus 
or drops at higher velocities. For $M<M_{e}$, the friction peak intensity decreases and broadens with $M$. The friction above $V_{o}$ reveals an unstable sliding process, Figure 5a, and the contact area shrinks from adhesive (JKR) to Hertzian, Figure 5b.

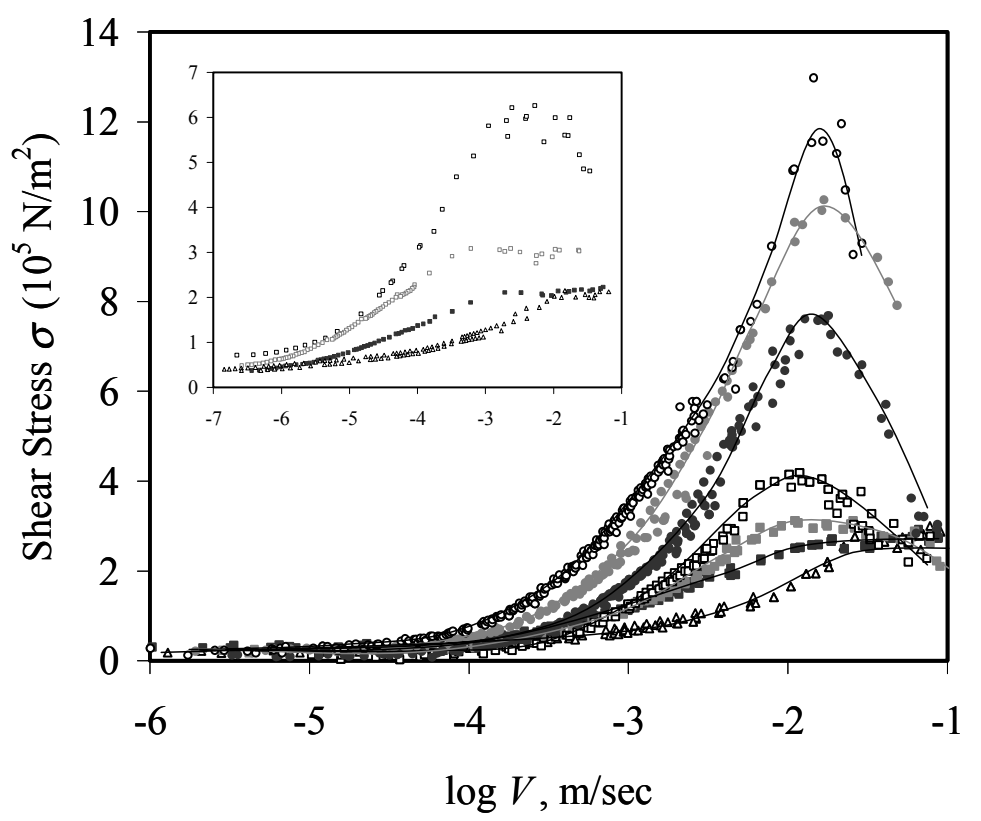

Figure 4. Shear stress as a function of velocity between PDMS and the SAM and (inset) PDMS and PS. ○, ॰, $\bullet, \square, \square, \boldsymbol{\square}$ and $\triangle$ represent networks with oligomeric precursors of $1.3,1.8,2.7,4.4,8.9,18.7$, and $52.1 \mathrm{~kg} / \mathrm{mol}$, respectively. $\left(M_{e} \sim 8 \mathrm{~kg} / \mathrm{mol}\right)$. Adapted from reference ${ }^{21}$.
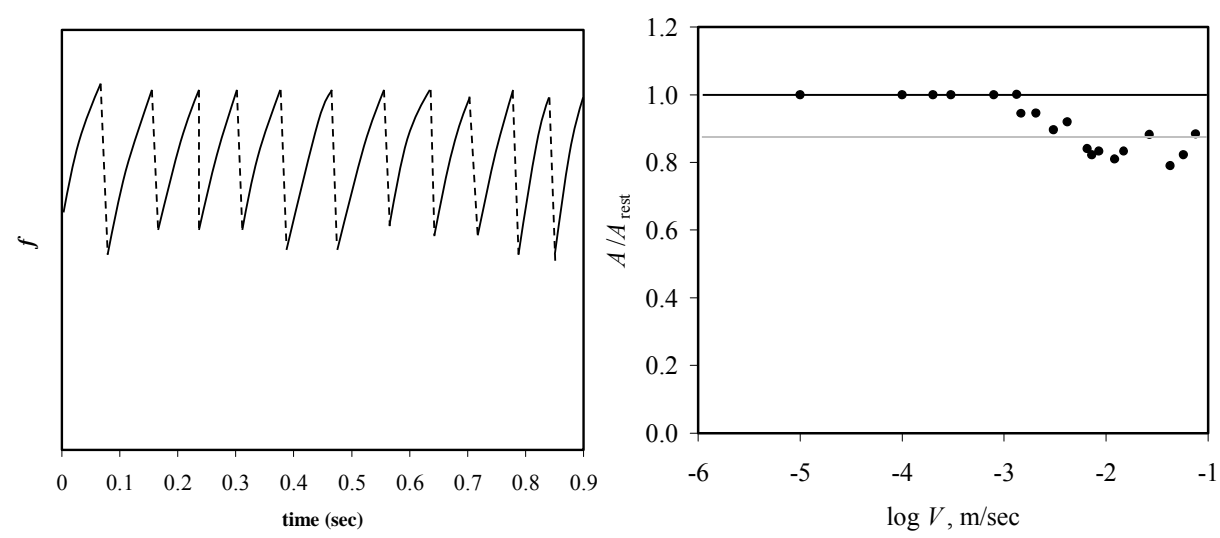

Figure 5. (a) A typical friction force trace for a PDMS hemisphere sliding across a methyl terminated SAM $(M=4.4 \mathrm{~kg} / \mathrm{mol}, V=2 \mathrm{~cm} / \mathrm{sec})$. Stick-slip sliding initiates at the critical velocity $V_{o}$ corresponding to the shear stress peak in Fig. 4. (b) Change in the sliding contact area A as a function of velocity for the PDMS /SAM interface, normalized by the contact area at rest $A_{\text {rest }}(M=2.7 \mathrm{~kg} / \mathrm{mol}, \mathrm{R}=2.5 \mathrm{~mm}, \mathrm{E}=4.8 \mathrm{MPa}, W=42$ $\mathrm{mJ} / \mathrm{mol}$ ). As the sliding velocity increases, the contact area drops from the JKR prediction (black line) to the purely Hertzian prediction (gray line). The normal load $F_{N}$ was constant at $48 \mathrm{mN}$ (see Equation 7). Adapted from reference ${ }^{21}$ 
These observations are qualitatively consistent with the stochastic theory for adhesive dominated friction, discussed above. Consider the inverse relationship between rubber friction and molecular weight in Figure 4, which has similarly been observed in other melts ${ }^{43,44}$ and grafted polymer chains. ${ }^{5,41}$ From a macroscopic perspective, Ludema and Tabor $^{25}$ suggested the relationship between the shear stress $\sigma$ and the areal density $\left(\Sigma_{\mathrm{o}}\right)$ of the contact points as $\sigma=\Sigma_{\mathrm{o}} f_{\mathrm{o}}$, where $f_{\mathrm{o}}$ is the adhesion force of a single polymer chain. This is similar to the prefactor in the Chernyak-Leonov equation (4) corresponding to the shear stress in the high velocity limit, i.e., where the detachment of the polymer chain from the surface is not stochastic. Within the simple model of Chernyak and Leonov ${ }^{23}$, the areal density of the load-bearing chains is $1 / N a^{2}$, where $N$ is the number of statistical segments, each of size $a$. Thus, the shear stress is proportional to the shear modulus as $\sigma=G \frac{f_{\circ} a}{k T}$. The areal chain density of a real elastomer; however, scales as $N^{1 / 2}$ and the shear stress should follow $\sigma \sim G^{1 / 2}$. Experimentally, Vorvolakos and Chaudhury observed $\sigma \sim G^{3 / 4}$. While Grosch $^{18}$ did not systematically consider the modulus, he noted that the shear stress is considerably smaller than that expected of two surfaces in true molecular contact and estimated the true contact area at roughly $10 \%$ of the apparent area during sliding. ${ }^{18}$ More recent SFM studies suggest that a gross mismatch of interfacial contact is not expected based on roughness considerations. Yet, it is plausible that spontaneous roughening of the interface occurs as a result of elastic instability, which ensues from the competition between van der Waals and elastic forces within the first layer of stretched PDMS chains in contact with the surface. If we consider that the dominant wavelength of such roughening scales with the thickness $(\delta)$ of the first layer of the polymer chain, then the density of the load bearing sites should scale as $1 / \delta^{2}$ (or $1 / N a^{2}$ ). In the limit of Rouse dynamics, if one polymer chain remains active in each of the load supporting junctions, one recovers: $\sigma \sim G$. The shear stress should decrease with the molecular weight owing to the fact that the number of load bearing polymer chains decreases with molecular weight. However, above the entanglement molecular weight, entanglement effects dominate the chain dynamics and $\sigma_{\max }$ becomes nearly independent of molecular weight.

\section{A Discussion in the Light of Molecular Relaxation within the Elastomer}

It turns out that by utilizing a molecular description, a cohesive process for frictional dissipation can be argued in favor of an adhesive one. Namely, as the molecular weight is increased (still below $M_{e}$ ) segmental relaxation times increase, indicating that deeper wells in the potential energy landscape restrict the degrees of freedom for molecular motion. For a given sliding velocity (or contact time), the molecules are less effective in dissipating energy internally through relaxation, and in accommodating the interface adhesively. Hence, friction and shear stress can be expected to decrease. Above $M_{e}$, the segmental relaxation time is essentially invariant with $M$, thus $F$ and $\sigma_{\max }$ are independent of $M$. It becomes apparent that the stochastic theory for adhesive dominated friction, applies almost in direct analogy to cohesive friction, by simply redefining $f_{o}$ as the internal friction force per molecule stretched in the contact (as shown in Figure 1). The same argument applies for the molecular weight, aerial chain density, modulus, and shear stress. Thus, again we are faced with a tribo- 
rheological complexity, where a distinction between adhesive and cohesive contributions to elastomeric friction require some characteristic signatures of the dissipation mechanism, e.g. the activation energy, relaxation time, or length scale of the process.

The characteristic signatures of the dissipation process can be determined by the superposition of friction-rate isotherms and from the critical velocity corresponding to the maximum in the friction force. ${ }^{18,21,45}$ This is illustrated in Figure 6 with superposed frictionvelocity isotherms obtained with SFM measurements on a polystyrene melt. ${ }^{45}$ An activation barrier of $81 \mathrm{kcal} / \mathrm{mol}(3.5 \mathrm{eV})$ is deduced from the apparent Arrhenius behavior of the thermal $a_{T}$ shift factor in the inset Fig. 6 . The value coincides with the $80-90 \mathrm{kcal} / \mathrm{mol}$ activation energy for the $\alpha$-relaxation process, ${ }^{46}$ i.e. the segmental relaxation of the PS backbone. In this case, the activation barrier overcome during the course of frictional sliding corresponds directly with the molecular relaxation within the bulk elastomer.

The friction peak is recognized as an analogue to a spectroscopic peak in the frequency space. ${ }^{18,45}$ It reflects the competition between material and experimental timescales. At low sliding velocities, the contact stress stored in the soft material is capable of relaxing (through internal friction modes) before an asperity can slip to the next contact site. In this region, the friction force increases logarithmically with velocity, which is consistent to an activated molecular relaxation process in the soft material. Above the critical velocity, the probing tip is driven to the next contact site before the material can respond internally through viscoelastic relaxation. Thus with increasing velocity, the tip experiences fewer and fewer dissipative relaxation events per jump, and consequently, the friction force decreases. Hence, molecular scale dynamics can be deduced from local friction measurements.

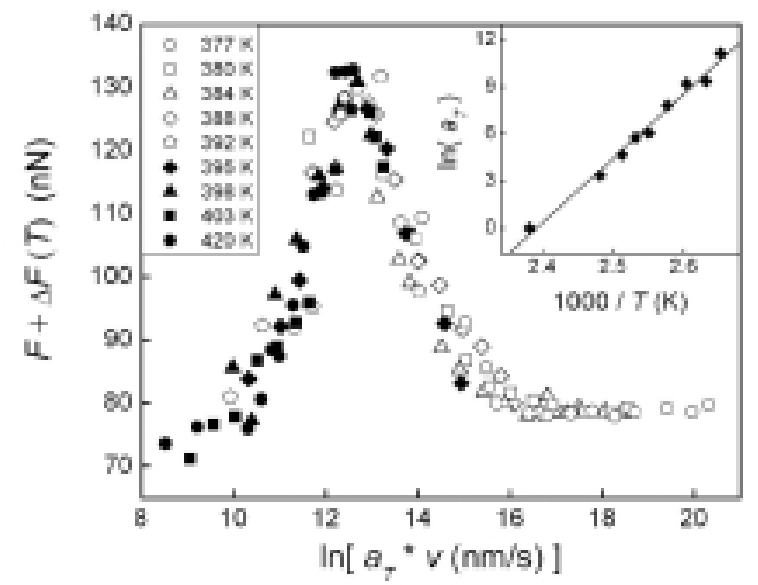

Figure 6. Friction force-velocity, $F(v)$ isotherms for polystyrene $(M=96.5 \mathrm{~kg} / \mathrm{mol})$ above $T_{g}=373 \mathrm{~K}$, superposed using the method of reduced variables with a reference temperature of $420 \mathrm{~K}$. Inset: From the Arrhenius behavior of $a_{T}$, an average activation energy, $E_{A}$, of $81 \mathrm{kcal} / \mathrm{mol}$ identifies the $\alpha$-relaxation as responsible for frictional dissipation. Adapted from reference ${ }^{45}$. 
A nanoscopic description of polymer dynamics involves, in general, only two parameters: an internal, or monomeric, friction coefficient and an appropriate macromolecular length scale. ${ }^{47}$ Internal friction dictates the degree of local segmental motion, and thus, is responsible for the bulk viscoelastic and relaxation properties of polymeric materials. Grosch ${ }^{18}$ and Ludema and Tabor ${ }^{48}$ were the first to combine the velocity at the friction peak with the frequency for the maximum viscoelastic loss to deduce a characteristic dissipative length scale. However, with their macroscopic techniques, they could only suspect dissipation through segmental relaxation. With the single asperity SFM approach to elastomeric friction, Sills, Gray and Overney, ${ }^{45}$ identified the specific molecular relaxation process activated in frictional sliding on an entangled polystyrene melt. With the velocity at the friction peak $V_{o}(T)$ in Figure $7 \mathrm{a}$ and knowledge of the associated relaxation times $\tau_{d}(T)$ from dielecrtic spectroscopy, they directly determined the dissipative length scale $X_{d}(T)$ of the $\alpha$-relaxation in a polystyrene melt via:

$$
X_{d}(T)=V_{o}(T) \cdot \tau_{\alpha}(T)
$$

The characteristic length of the dissipation process was found to grow from the segmental scale to $2.1 \mathrm{~nm}$, following a power law behavior that is consistent with predictions for cooperative motion during the $\alpha$-relaxation, Figure $7 \mathrm{~b}$. However, in the vicinity of the glass transition, $T_{g}=373 \mathrm{~K}$, dissipation lengths of several tens of nanometers deviate from the above power law behavior, suggesting that long-range processes, i.e. Fischer modes, may couple with the $\alpha$-relaxation with an apparent non-ergodic time-averaged behavior.

In the inset of Figure 7a, the friction-peak drops sharply as the melt is cooled toward $T_{g}$, indicating that the number of $\alpha$-relaxation events per jump decreases with the onset of vitrification. Considering, as discussed above, that frictional dissipation on a glassy polystyrene surface is dominated by the lower energy side-chain relaxation, the attenuation of the $\alpha$-friction-peak intensity in the crossover regime $\left(T_{g}<T<T_{c}\right)$ reflects a structurally and dynamically heterogeneous phase, with a spatial distribution of dissipation through independent backbone and side-chain relaxations. One would then expect a simultaneous increase in a $\beta$-friction peak at a velocity corresponding to the time scale for side chain rotation. Unfortunately, the frequency (i.e. velocity) bandwidth of the SFM is not sufficient to capture the fast rotational peak of the phenyl rings, which would be expected at a velocity on the order of centimeters per second. 

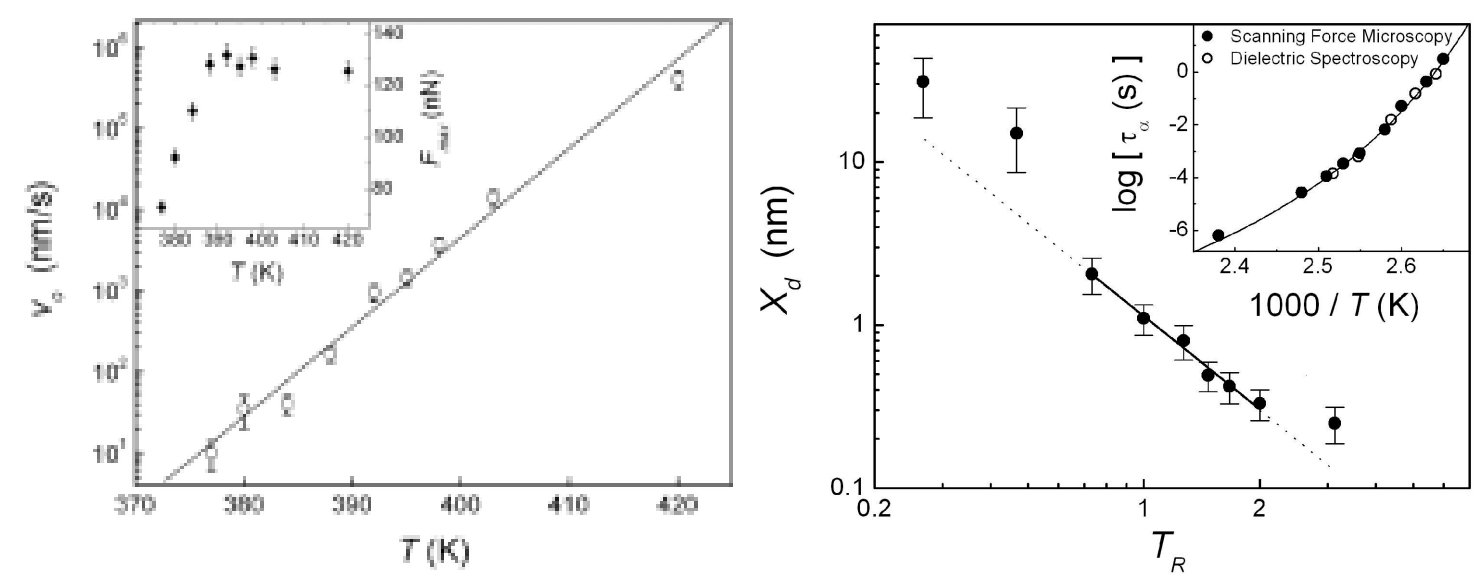

Figure 7. (a) the velocity $v_{o}$ corresponding to the friction peak of polystyrene $(M=96.5 \mathrm{~kg} / \mathrm{mol}$, $M_{e} \sim 20 \mathrm{~kg} / \mathrm{mol}$ ) in Figure 6. (inset) The corresponding friction peak intensities $F_{\max }$. (b) Dissipation length $X_{d}$ for the $\alpha$-relaxation of polystyrene in terms of the reduced temperature, $T_{R}=\left(T-T_{g}\right) /\left(T_{c}-T_{g}\right)$ where $T_{g}$ is the calorimetric glass transition temperature $(373 \mathrm{~K})$ and $T_{c}$ is the crossover temperature of the dynamic glass transition $(388 \mathrm{~K})$ identified at the saturation point of $F_{\max }$ in the inset of (a). The power law fit $X_{d} \sim T_{R}^{-\phi}$ over the range $0.7<T_{R}<2.0$ (solid line) reveals an exponent of $\phi=1.89 \pm 0.08$. (inset) polystyrene $\alpha$-relaxation times from dielectric spectroscopy ${ }^{49}$ (open circles) and the corresponding times from $V_{o}$ and $X_{d}$ and equation 8 (closed circles). Adapted from reference ${ }^{45}$.

\section{Dissipation due to Decoupling of Adhesive Bonds versus Segmental Relaxations}

The work of Vorvolakos and Chaudhury ${ }^{21}$ specifically targeted a decoupling of the adhesive and cohesive components of elastomeric friction. The internal friction associated with the elastomer (PDMS hemispheres) was varied through the crosslink density (i.e. molecular weight between crosslinks $M_{c}$ ), and the interfacial adhesion was catered via the surface energy of the counter-faces: a methyl terminated hexadecylsiloxane self-assembled monolayer (SAM) and a thin film of glassy polystyrene (PS). While both surfaces are essentially non-polar, the critical velocity at the friction peak $V_{o}$ differs by almost an order of magnitude between the SAM and PS surfaces (Figure 4). On the other hand, $V_{o}$ is independent of the intercrosslink molecular weight, above and below the entanglement weight. Initially, a strong adhesive contribution is suspected; however, a shift in $V_{o}$ may be attributed to a change in the timescale of the dissipation process, a change in the lengthscale over which dissipation occurs, or both in the event of a completely different dissipation mechanism.

For adhesive dominated friction, the critical velocity, according to Chernyak and Leonov, appears at $V_{\circ}=\frac{\delta \cot \chi}{\tau_{\circ}}$. As $\delta \sim N^{1 / 2} a$ and $\cot \chi \sim \frac{f_{\circ} \delta}{k T}, V_{\circ} \sim \frac{f_{\circ} N a^{2}}{k T \tau_{\circ}}$. It was argued by Chernyak and Leonov that the life time $\tau_{0}$ of the bound state of the polymer chain at rest is related to the characteristic time of $\tau$ segmental motion as $\tau_{\circ}=\tau\left(R_{F} / a\right)^{3}$, which results in the molecular velocity $V_{\mathrm{o}}$ decreasing with $N$ following a $1 / 2$ power law. However, the experimental data of Vorvolakos and Chaudhury ${ }^{21}$ indicate that $V_{\mathrm{o}}$ is nearly independent of molecular weight for PDMS $(M \alpha N)$, thus suggesting that $\tau_{0}$ varies linearly with $N$, not as $N^{3 / 2}$. 
If dissipation through segmental relaxation is considered, the segmental length of PDMS $(0.6 \mathrm{~nm})$ combined with the friction peak velocities in Figure 4, gives characteristic times of $10^{-7}$ and $10^{-8} \mathrm{~s}$, for the SAM and PS surfaces, respectively. These times are considerably larger than the viscous relaxation time $\left(10^{-11} \mathrm{~s}\right)$ of dimethylsiloxane monomer, ${ }^{50}$ however, the relaxation dynamics in crosslinked PDMS occur on different time scales. The fast mode that follows from the Rouse-like dynamics of chain segments between entanglements occurs on a time scale of $10^{-9} \mathrm{~s}$ at room temperature. ${ }^{51}$ On the other hand, the slow mode translational dynamics of crosslink junctions through the polymer network occurs at $10^{-7} \mathrm{~s}$, independent of molecular weight. ${ }^{51}$ The correspondence of the PDMS friction peak velocity with the slow mode dynamics suggests dissipation through crosslink motion. Interestingly, junction fluctuation theories predict that the mobility of crosslink junctions is of crucial importance in determining the modulus of the network. $^{51}$

The dissipation picture is still not complete. The shift in the friction peak velocity due to the surface energy of the opposing SAM or PS surfaces implies a significant adhesive contribution. By the superposition of friction-rate isotherms $\left(T>T_{g}=-130{ }^{\circ} \mathrm{C}\right)$, Vorvolakos and Chaudhury $^{21}$ were able to estimate Arrhenius activation energies of 25 and $27 \mathrm{~kJ} / \mathrm{mol}$ for dissipation on the SAM and PS surfaces, respectively. While the activation energies did not differ substantially, the pre-exponential factors varied by one order of magnitude, from $10^{-12}$ to $10^{-11}$ for the SAM and PS surfaces, respectively. The activation energies are considerably higher than that for the bulk viscosity of PDMS $(16 \mathrm{~kJ} / \mathrm{mol})$, which suggest that the potential barrier overcome during the course of frictional sliding may be modified by the adhesive interactions. However, fluorescent probe reorientation measurements in PDMS have revealed activation energies of $20-28 \mathrm{~kJ} / \mathrm{mol}$ for both the fast and slow relaxation modes. ${ }^{52}$ The barrier enhancement relative to the bulk viscosity was attributed to the unique nature of the silicon-oxygen backbone, which results in a local mobility that is quite distinct from the long-range chain dynamics. Thus, the observed barrier height during frictional sliding is sufficient to activate viscoelastic relaxation in the PDMS elastomer, presumably the slow mode associated with crosslink mobility. For this case, the adhesive contribution appears, not strictly in the barrier height, but in the barrier jump attempt frequency, i.e. in the exponential pre-factor in the Arrhenius equation. This suggests that the intrinsic chemical interactions at the contact interface dictate the lifetime of the bound state at rest, i.e. $\tau_{o}$ in equation 2 , for adhesive debonding.

\section{Outlook and Closing Remarks}

We have discussed the molecular origins of elastomeric friction in the light of two different processes: interfacial adhesion and internal friction. Various studies have highlighted the importance of both processes; however, a unified picture that accurately accounts for both has yet to evolve. On the molecular scale bulk rheological constructs like viscosity and modulus are insufficient to capture the interfacial molecular dynamics. Both processes are rate dependent, and both can be described by Eyring's activation model. While most studies have focused on one or the other aspect, it is unlikely that the two processes are mutually independent. A more realistic picture considers the competition between the two. Resolution of the debate is likely to unfold by considering the energetic signatures of each process. We should ask what potential barrier is higher, adhesive bonding-debonding, or internal molecular relaxation of the stressed elastomer. If interfacial adhesion dominates internal cohesion, sliding asperities are apt to be coated with a molecular layer of the elastomer, forming a sliding plane within the bulk elastomer 
and yielding frictional properties related to the bulk material properties. On the other hand, if internal cohesion dominates interfacial adhesion, like in many rigid materials within limits of plastic yield, the frictional outcome is likely to reflect the interfacial interactions.

As we move into the new millennium, precise understanding of the frictional process is necessary for the development of advanced materials. A refined understanding of molecular friction is critical to emerging nanotechnologies, e.g., nano-electromechanical systems $(\mathrm{NEMS})^{39,53}$ and biomaterials. ${ }^{54-56}$ On this scale, all transport processes depend on the degrees of freedom available to molecular motion.

\section{References}

1 R. Ariano, The Coefficients of Friction Between Rubber \& Various Materials. Part II - Gripping Friction of Rubber Belting, India Rubber Journal 79(2), 56-8 (1930).

2 J. B. Derieux, The Coefficients of Friction Between Rubber \& Various Materials. Part II - Gripping Friction of Rubber Belting, J. Elisha Mitchell Scientific Society 50, 53-5 (1934).

3 T. R. Dawson and B. D. Porritt, Rubber: Physical and Chemical Properties, 381$386(1935)$.

4 B. Z. Newby, M. K. Chaudhury, and H. R. Brown, Macroscopic Evidence of the Effect of Interfacial Slippage on Adhesion, Science 269, 1407-8 (1995).

5 B. Newby and M. K. Chaudhury, Friction in Adhesion, Langmuir 14, 4865-90 (1998).

6 S. L. Aggarwal, I. G. Hargis, R. A. Livigni, H. J. Fabris, and L. F. Marker, in Advances in Elastomers and Rubber Elasticity (Plenum, New York, 1986).

7 A. N. Theodore, M. A. Samus, and P. C. Killgoar, Environmentally Durable Elastomer Materials for Windshield Wiper Blades, Ind. Eng. Chem. Res. 31 (12), 2759-64 (1992).

8 C. W. Extrand, A. N. Gent, and S. Y. Kaang, Friction of a rubber wedge sliding on glass, Rubber Chemistry and Technology 64 (1), 108-17 (1991).

9 F. L. Roth, R. L. Driscoll, and W. L. Holt, Frictional Properties of Rubber, J. Research of the National Bureau of Standards 28(4), 439-62 (1942).

10 P. Thirion, Les coefficients d' adherence du caoutchouc., Revue Generale du Caoutchouc 23(5), 101-6 (1946).

11 P. J. Papenhuyzen, Wrijvingsproeven in verband met het slippen van autobanden, De Ingenieur V75, 53 (1938).

12 A. Schallamach, The Velocity and Temperature Dependence of Rubber Friction, Proceedings of the Physical Society B 65, 657-61 (1952).

13 A. Schallamach, The load dependence of rubber friction, Proceedings of the Physical Society B 66, 386-92 (1952).

14 F. P. Bowden and D. Tabor, The Friction and Lubrication of Solids (Oxford University Press, 1950).

15 H. Eyring, Viscosity, plasticity, and diffusion as examples of absolute reaction rates, J. Chemical Physics 4, 283-91 (1936). 
16 J. A. Greenwood and D. Tabor, The Friction of Hard Sliders on Lubricated Rubber: The Importance of Deformation Losses, Proceedings of the Physical Society 71, 989-1001 (1958). A. M. Bueche and D. G. Flom, Surface friction and dynamic mechanical properties of polymers, Wear 2 (3), 168-82 (1959).

$18 \mathrm{~K}$. A. Grosch, The relation between the friction and visco-elastic properties of rubber, Proceedings of the Royal Society A 274, 21-39 (1963).

19 M. L. Williams, R. F. Landel, and J. D. Ferry, The Temperature Dependence of Relaxation Mechanisms in Amorphous Polymers and other Glass-forming Liquids, J. Am. Chem. Soc. 77, $3701-7$ (1955).

20 A. Schallamach, A theory of dynamic rubber friction, Wear 6, 375-82 (1963).

21 K. Vorvolakos and M. K. Chaudhury, The effects of molecular weight and temperature on the kinetic friction of silicone rubbers, Langmuir 19, 6778-87 (2003).

22 T. Kontorova and Y. I. Frenkel, On the theory of plastic deformation and twinning, Soviet Phys. JEPT 13, 1-10 (1938).

23 Y. B. Chernyak and A. I. Leonov, On the Theory of Adhesive Friction of Elastomers, Wear 108, 105-38 (1986).

24 A. R. Savkoor, On the friction of rubber, Wear 8, 222-37 (1965).

25 K. C. Ludema and D. Tabor, The friction and visco-elastic properties of polymeric solids, Wear 9, 329-48 (1966).

26 A. Schallamach, How does rubber slide?, Wear 17, 301-12 (1971).

27 A. D. Roberts and S. A. Jackson, Sliding friction of rubber, Nature 257, 118-20 (1975).

28 J. I. Siepmann and I. R. McDonald, Monte Carlo Simulation of the Mechanical Rexation of a Self-Assembled Monolayer, Phys. Rev. Lett. 70, 453-6 (1993).

29 T. Bonner and A. Baratoff, Molecular dynamics study of scanning force microscopy on self-assembled monolayers, Surf. Sci. 377-379, 1082-6 (1997).

30 P. T. Mikulski and J. A. Harrison, Packing-density effects on the friction of nalkane monolayers, J. Am. Chem. Soc. 123, 6873-81 (2001).

31 R. M. Overney, H. Takano, M. Fujihira, W. Paulus, and H. Ringsdorf, Anisotropy in friction and molecular stick-slip motion, Phys. Rev. Lett. 72, 3546-49 (1994).

32 A. Ulman, An Introduction to Ultrathin Organic Films (Academic Press, New York, 1991).

33 G. A. Tomlinson, Philos. Mag. Ser. 7, 905 (1929).

34 O. K. Dudko, A. E. Filippov, J. Klafter, and M. Urbakh, Dynamic force spectroscopy: a Fokker-Planck approach, Chem. Phys. Lett. 352, 499-504 (2002).

35 Y. Sang, M. Dube, and M. Grant, Thermal Effects on Atomic Friction, Phys. Rev. Lett. 87, 174301/1-4 (2001).

36 F. Heslot, T. Baumberger, B. Perrin, B. Caroli, and C. Caroli, Creep, stick-slip, and dry-friction dynamics: experiments and a heuristic model, Phys. Rev. E 49, 4973-88 (1994).

37 S. Sills and R. M. Overney, Creeping friction dynamics and molecular dissipation mechanisms in glassy polymers, Phys. Rev. Lett. 91, 095501(1-4) (2003). 
38 J. A. Hammerschmidt, W. L. Gladfelter, and G. Haugstad, Probing polymer viscoelastic relaxations with temperature-controlled friction force microscopy, Macromolecules 32, 3360-7 (1999).

39 B. Gotsmann, S. Sills, U. Duerig, J. Frommer, and C. Hawker, Controlling nanowear in a polymer by confining segmental relaxation, NanoLett. (2006).

40 H. R. Brown, Chain Pullout and Mobility Effects in Friction and Lubrication, Science 263, 1411-3 (1994).

41 A. Casoli, M. Brendle, J. Schultz, A. Philippe, and G. Reiter, Friction Induced by Grafted Polymeric Chains, Langmuir 17 (2), 388-98 (2001).

42 A. Ghatak, K. Vorvolakos, H. She, D. Malotky, and M. K. Chaudhury, Interfacial Rate Processes in Adhesion and Friction, J. Physical Chemistry B 104 (17), 401830 (2000).

43 Y. Inn and S.-Q. Wang, Hydrodynamic slip: Polymer adsorption and desorption at melt/solid interfaces, Physical Review Letters 76 (3), 467-70 (1996).

44 S. Hirz, A. Subbotin, C. Frank, and G. Hadziioannou, Static and kinetic friction of strongly confined polymer films under shear, Macromolecules 29 (11), 3970-4 (1996).

45 S. Sills, T. Gray., and R. M. Overney, Molecular dissipation phenomena of nanoscopic friction in the heterogeneous relaxation regime of a glass former, $\mathrm{J}$. Chem. Phys. 123, 134902 (2005).

46 G. D. Patterson, C. P. Lindsey, and J. R. Stevens, Depolarized Rayleigh spectroscopy of polystyrene near the glass-rubber transition, J. Chem. Phys. 70, 643-5 (1979).

47 V. N. Pokrovskii, Mesoscopic theory of polymer dynamics (Kluwer Academic Publishers, Dordrecht, 2000).

48 K. C. Ludema and D. Tabor, The friction and visco-elastic properties of polymer solids, Wear 9, 329-48 (1966).

49 C. M. Roland and R. Casalini, Temperature dependence of local segmental motion in polystyrene and its variation with molecular weight, J. Chem. Phys. 119, 1838-42 (2003).

50 M. Appel and G. Fleischer, Investigation of the chain length dependence of selfdiffusion of poly(dimethylsiloxane) and poly(ethylene oxide) in the melt with pulsed field gradient NMR, Macromolecules 26, 5520-5 (1993).

51 P. B. Leezenburg, M. D. Frayer, and C. W. Frank, Photophysical studies of probes bound to crosslinked junctions in poly(dimethyl silozane) elastomers and nanocomposites, Pure \& Appl. Chem. 68, 1381-8 (1996).

52 A. D. Stein, D. A. Hoffmann, A. H. Marcus, P. B. Leezenburg, and C. W. Frank, Dynamics in Poly(dimethylsiloxane) Melts: Flouresence Depolarization Measurements of Probe Chromophore Orientational Relaxation, J. Phys. Chem 96, 5255-63 (1992).

53 S. E. Sills, R. M. Overney, B. Gotsmann, and J. Frommer, Strain shielding and confined plasticity in thin polymer films: Impacts on thermomechanical data storage, Tribo. Lett. 19, 9-15 (2005).

54 H. B. Dong, T. Blawert, C. Mordike, B. L., Plasma immersion ion implantation of UHMWPE, J. Mater. Sci. Lett. 19 (13), 1147-9 (2000). 
55 J. Wang, E. Stroup, X. Wang, and A. J. D., Study of PEO on LTI Carbon Surfaces by Ellipsometry and Tribometry, Proc. SPIE-Int. Soc. Opt. Eng. Int. Conf. Thin Film Phys. Appl. Pt. 2, 835-41 (1991).

56 T. Murayama and C. R. McMillin, Dynamic mechanical properties of elastomers for use in circulatory assist devices, J. Appl. Polym. Sci. 28 (6), 1871-7 (1983). 